\title{
Static Analysis of Stiffened Shells Using an Edge-Based Smoothed MITC3 (ES-MITC3) Method
}

\author{
Quoc Hoa Pham (iD) and Phu-Cuong Nguyen \\ Advanced Structural Engineering Laboratory, Faculty of Civil Engineering, Ho Chi Minh City Open University, \\ Ho Chi Minh City, Vietnam \\ Correspondence should be addressed to Quoc Hoa Pham; hoa.pq@ou.edu.vn
}

Received 12 May 2021; Accepted 26 August 2021; Published 22 September 2021

Academic Editor: Francesco Foti

Copyright (c) 2021 Quoc Hoa Pham and Phu-Cuong Nguyen. This is an open access article distributed under the Creative Commons Attribution License, which permits unrestricted use, distribution, and reproduction in any medium, provided the original work is properly cited.

\begin{abstract}
A novel approach for solving the stiffened shell structures by using an edge-based smoothed MITC3 finite element method (ESMITC3) is presented in this paper. The ES-MITC3 method is an efficient finite element method by combining the edge-based smoothed finite element method (ES-FEM) with the original MITC3 triangular element to not only significantly improve the accuracy but also overcome the shear-locking phenomenon in the Reissner-Mindlin shell analysis. In this study, the ES-MITC3 method is applied for shell structures and then reinforced by stiffeners based on the Timoshenko beam theory to achieve more durability and strength structures. The transverse displacements of the shell structures and stiffeners at the contact positions are assumed compatible. Numerical results of the ES-MITC3 element are compared with those of available other numerical results to demonstrate a good convergence and accuracy of the present method.
\end{abstract}

\section{Introduction}

Many engineering structures based on plate/shell models are often reinforced by stiffeners, named stiffened plates/shells, to achieve more durability and strength $[1,2]$. Hence, the study of stiffened shell structures has unendingly received heavy interest from many researchers over the world in recent decades.

For analytical methods, Mecitoğlu [3] firstly presented a free vibration analysis of stiffened conical thin shells based on Donnell-Mushtari theory. The evaluation of free vibration of cylindrical stiffened shells with rings and stringers by using and comparing Donnell's, Love's, Sanders', and Flugge's thin shell theories was addressed by Ruotolo [4]. Pan et al. [5] developed the classical thin shell theory [6] to investigate free vibration of cylindrical stiffened shells with arbitrary boundary conditions. Nevertheless, the analytical methods are difficult to analyze for stiffened shells due to the complexity of structural shapes in practice. As a result, numerical methods are preferred for solving the stiffened plates/shells, especially the finite element (FE) methods.
In analyzing the stiffened shell structures using FE methods, a favored and popular approach is separating the stiffened shell structures into two parts: a background shell element and stiffener elements while maintaining the monolithic structure state. Palani et al. [7] introduced eightand nine-node isoparametric plate/shell elements combined with three-node isoparametric beam elements for static and vibration analyses of the stiffened plates/shells. An investigating behavior of stiffened laminated cylindrical shells is proposed by Venkatesh and Rao [8]. In this work, a four-node laminate thin shell element using 48 degrees of freedom (d.o.f) is incorporated with an anisotropic beam element of 16 d.o.f. Liao and Reddy [9] developed a threedimensional continuum shell element combining with curved beam elements to investigate the geometric nonlinear analysis of laminated stiffened shells. In order to solve the elastic static behavior of isotropic stiffened shells, Prusty and Satsangi [10] combined a three-node beam element for the stiffeners and an eight-node quadrilateral element for the shell structures. Using a shallow shell model, Sinha et al. [11] proposed a stiffened triangular shell element with 36 d.o.f in 
which the stiffener elements are placed anywhere in the shell elements.

As discussed above, almost FE methods proposed for analyzing stiffened shell structures used higher-order elements based on the degenerated shell or shallow thin shell theories. These kinds of elements require a large number of d.o.f, which leads to complicated analysis and high computational cost. As an alternative method, a Reissner-Mindlin plate/shell theory combined flat shell models can provide a simpler derivation and promising method for solving the stiffened shell structures. Samanta and Mukhopadhyay [2,12] incorporated the Allman and DKT plate elements for the geometric nonlinear static and vibration analysis of stiffened shells. Nguyen et al. [13,14] developed the cell-based smoothed discrete shear gap method (CS-DSG3) to investigate for static, free vibration, and buckling of stiffened plates/shells.

Recently, in order to improve the accuracy of the original MITC3 triangular element, an efficient method by combining the edge-based smoothed finite element method (ESFEM) [15-20] and the MITC3 triangular element, so-called ES-MITC3 [21-25], is recently presented. The numerical results are conducted by these studies show that the ESMITC3 is almost found to achieve better convergence and accuracy than the original MITC3 triangular element, and existing free shear-locking shell elements such as DSG3 [26], MITC3 [27], and MITC4 [28].

This paper now extends the application of ES-MITC3 to the static analysis of the stiffened shells by incorporating the shell element ES-MITC3 and a linearly isotropic two-node Timoshenko beam element, respectively. In this work, we prove that the triangular ES-MITC3 element is a strong competitor to other existing shell elements in the literature.

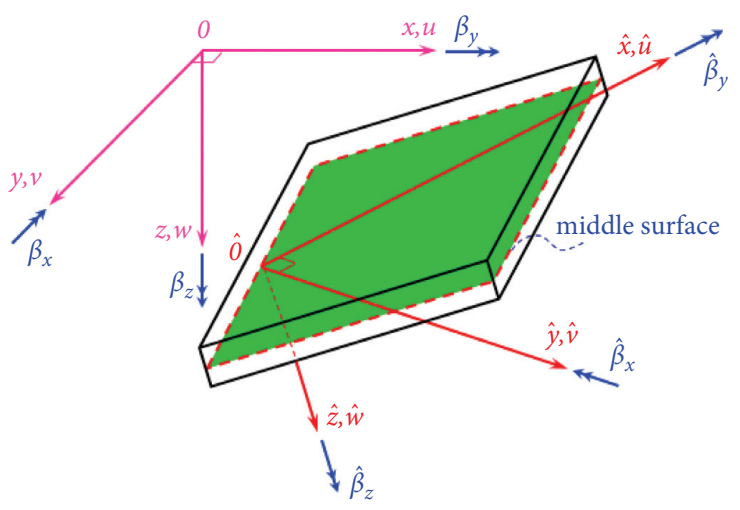

FIgURE 1: A shell element model in the local coordinate $\widehat{O} \hat{x} \hat{y} \widehat{z}$ and global system $O x y z$.

\section{A Brief on the ES-MITC3 Formulation for the Stiffened Shell}

A flat shell structural element with the global coordinate $O x y z$ and local coordinate $\widehat{O} \hat{x} \widehat{y} \widehat{z}$ systems is shown in Figure 1. Let $\left(\widehat{u}, \widehat{\beta}_{x}\right),\left(\widehat{v}, \widehat{\beta}_{y}\right)$ and $\left(\widehat{w}, \widehat{\beta}_{z}\right)$ are the rotations and displacements at the middle surface of the shell element in the $\hat{x}, \hat{y}$, and $\widehat{z}$ directions, respectively. The unknown nodal vector of displacement field with six independent variables can be given as follows:

$$
\widehat{u}^{T}=\left\{\widehat{u}, \widehat{v}, \widehat{w}, \widehat{\beta}_{x}, \widehat{\beta}_{y}, \widehat{\beta}_{z}\right\} .
$$

In the well-known Reissner-Mindlin shell formulation, the membrane strain $\widehat{\varepsilon}_{m}$, the bending strain $\widehat{\kappa}$, and the shear strains $\widehat{\gamma}$ in the local coordinate system $\widehat{O} \widehat{x} \widehat{y} \widehat{z}$ are defined, respectively, by

$$
\begin{aligned}
& \widehat{\varepsilon}_{m}=\left\{\begin{array}{c}
\frac{\partial \widehat{u}}{\partial \hat{x}} \\
\frac{\partial \widehat{v}}{\partial \hat{y}} \\
\frac{\partial \widehat{u}}{\partial \hat{y}}+\frac{\partial \widehat{v}}{\partial \hat{x}}
\end{array}\right\}=\mathbf{L}_{m} \widehat{u}, \quad \text { with } \mathbf{L}_{m}=\left[\begin{array}{cccccc}
\frac{\partial}{\partial \widehat{x}} & 0 & 0 & 0 & 0 & 0 \\
0 & \frac{\partial}{\partial \hat{y}} & 0 & 0 & 0 & 0 \\
\frac{\partial}{\partial \hat{y}} & \frac{\partial}{\partial \hat{x}} & 0 & 0 & 0 & 0
\end{array}\right], \\
& \widehat{\kappa}=\left\{\begin{array}{c}
\frac{\partial \hat{\beta}_{x}}{\partial \hat{x}} \\
\frac{\partial \widehat{\beta}_{y}}{\partial \widehat{y}^{2}} \\
\frac{\partial \hat{\beta}_{x}}{\partial \hat{y}}+\frac{\partial \widehat{\beta}_{y}}{\partial \hat{x}}
\end{array}\right\}=\mathbf{L}_{b} \widehat{u}, \quad \text { with } \mathbf{L}_{b}=\left[\begin{array}{cccccc}
0 & 0 & 0 & \frac{\partial}{\partial \hat{x}} & 0 & 0 \\
0 & 0 & 0 & 0 & \frac{\partial}{\partial \hat{y}} & 0 \\
0 & 0 & 0 & \frac{\partial}{\partial \hat{y}} & \frac{\partial}{\partial \hat{x}} & 0
\end{array}\right], \\
& \widehat{\gamma}=\left\{\begin{array}{l}
\frac{\partial \widehat{w}}{\partial \widehat{x}}+\widehat{\beta}_{\widehat{x}} \\
\frac{\partial \widehat{w}}{\partial \hat{y}}+\widehat{\beta}_{\widehat{y}}
\end{array}\right\}=\mathbf{L}_{s} \widehat{u}, \quad \text { with } \mathbf{L}_{s}=\left[\begin{array}{cccccc}
0 & 0 & \frac{\partial}{\partial \widehat{x}} & 1 & 0 & 0 \\
& & & & & \\
0 & 0 & \frac{\partial}{\partial \hat{y}} & 0 & 1 & 0
\end{array}\right] .
\end{aligned}
$$


The shell structures are reinforced by stiffeners to achieve more durability and strength with a minimal weight increase. In general, the stiffeners are represented by the beam structures placed parallel to the edges of shell structures. It should be noted that the phenomenon of warping with beams is not considered in our study [14]. Figure 2 shows a stiffener placed askew an angle $\phi$ to the $\widehat{x}$-axis of the flat shell element, wherein the plane $\widehat{O} \widehat{r} \widehat{s}$ in the local coordinate $\widehat{O} \widehat{r} \widehat{s} \hat{z}$ of the stiffener and the plane $\widehat{O} \hat{x} \hat{y}$ in the local coordinate $\widehat{O} \hat{x} \hat{y} \widehat{z}$ of the flat shell element are assumed to be coincident [29]. As presented in [14], the elastic strain field of a stiffener in local coordinate $\widehat{O} \hat{x} \widehat{y} \widehat{z}$ based on the Timoshenko beam theory is given as

$$
\widehat{\varepsilon}_{\mathrm{st}}=\mathbf{n} \cdot \nabla\left[\begin{array}{ccccc}
n_{x} & n_{y} & 0 & e n_{x} & e n_{y} \\
0 & 0 & 0 & n_{x} & n_{y} \\
0 & 0 & 1 & \frac{n_{x}}{\mathbf{n} \cdot \nabla} & \frac{n_{y}}{\mathbf{n} \cdot \nabla} \\
0 & 0 & 0 & -n_{y} & n_{x}
\end{array}\right] \underbrace{\left[\begin{array}{c}
\widehat{u}_{\mathrm{st}} \\
\widehat{v}_{\mathrm{st}} \\
\widehat{w}_{\mathrm{st}} \\
\widehat{\beta}_{\mathrm{st} \cdot x} \\
\widehat{\beta}_{\mathrm{st} \cdot y}
\end{array}\right]}_{\mathbf{L}_{\mathrm{st}}}=\mathbf{L}_{\mathrm{st}} \widehat{u}_{\mathrm{st}},
$$

where $\mathbf{n}=\left\{n_{x}, n_{y}\right\}$ is the direction cosine vector, $e$ is the eccentricity between the middle surface of the flat shell element and the centroid of the stiffener as plotted in Figure 2, and $\widehat{u}_{s t}=\left[\widehat{u}_{\text {st }}, \widehat{v}_{\text {st }}, \widehat{w}_{\text {st }}, \widehat{\beta}_{\text {st } x}, \widehat{\beta}_{\text {st. } y}\right]^{T}$ is the displacement fields of the stiffener in the local coordinate $\widehat{O} \widehat{x} \hat{y} \widehat{z}$. It should be noted that the transverse displacements of the shell structures and stiffeners at the contact positions are assumed compatible in this study [14,29]. Then, the displacement fields of the shell structure and stiffeners $\left(\widehat{u}, \widehat{u}_{s t}\right)$ in the local coordinate $\widehat{O} \hat{x} \widehat{y} \widehat{z}$ are transformed to $\left(\mathbf{u}, \mathbf{u}_{s t}\right)$ in the global coordinate $O x y z$ by the transformation matrix $\Lambda$ presented in detail in $[21,23]$.

By using Hamilton's principle, the weak form for the static analysis of the Reissner-Mindlin shell reinforced by a stiffener with a problem domain $\Omega \subset \mathbb{R}^{3}$ can be established as [14]

$$
\iint_{\Omega} \delta \mathbf{u}^{T}\left(\mathbf{L}_{m}^{T} \mathbf{D}_{m} \mathbf{L}_{m}+\mathbf{L}_{b}^{T} \mathbf{D}_{b} \mathbf{L}_{b}+\mathbf{L}_{s}^{T} \mathbf{D}_{s} \mathbf{L}_{s}\right) \mathbf{u d} \Omega+\int_{l} \delta \mathbf{u}_{\mathrm{st}}^{T} \mathbf{L}_{\mathrm{st}} \mathbf{D}_{\mathrm{st}} \mathbf{L}_{\mathrm{st}} \mathbf{u}_{\mathrm{st}} \mathrm{d} l=\iint_{\Omega} \delta \mathbf{u}^{T} \mathbf{b} \mathrm{d} \Omega
$$

where $\mathbf{b}$ is the body force of the shell and $\mathbf{D}_{m}, \mathbf{D}_{b}, \mathbf{D}_{s}$, and $\mathbf{D}_{s t}$ are the material matrices given, respectively, as follows:

$$
\begin{aligned}
& \mathbf{D}_{m}=\frac{E h}{1-v^{2}}\left[\begin{array}{ccc}
v & 1 & 0 \\
0 & 0 & \frac{1-v}{2}
\end{array}\right], \\
& \mathbf{D}_{b}=\frac{E h^{3}}{12\left(1-v^{2}\right)}\left[\begin{array}{ccc}
1 & v & 0 \\
v & 1 & 0 \\
0 & 0 & \frac{1-v}{2}
\end{array}\right], \\
& \mathbf{D}_{s}=k h G\left[\begin{array}{ll}
1 & 0 \\
0 & 1
\end{array}\right], \\
& \mathbf{D}_{\mathrm{st}}=\operatorname{diag}\left(E A_{\mathrm{st}}, E I_{s}, k G A_{\mathrm{st}}, G J\right),
\end{aligned}
$$

in which $G, k=(5 / 6), h, E$, and $v$ are the shear modulus, shear correction factor, the thickness of the shell, Young's modulus, and Poisson's ratio, respectively. In equation (6), $A_{\text {st }}, I_{s}$, and $J$ are the cross area, second moment of the crosssectional area, and the torsional modulus of the stiffener, respectively, as expressed in [14].

Next, the finite element (FE) approximation for weak form (4) can be expressed as follows [14]:

$$
\mathbf{K} \mathbf{d}=\mathbf{F} \text {, }
$$

where $\mathbf{K}, \mathbf{F}$, and $\mathbf{d}$ are the global stiffness matrix, load vector, and the displacement vector in global coordinate $O x y z$, respectively. The global stiffness matrix $\mathbf{K}$ in equation (7) is computed based on a combination between the global stiffness matrix of shell structure $\mathbf{K}_{\mathrm{sh}}$ and stiffeners $\mathbf{K}_{\mathrm{st}}$ as

$$
\mathbf{K}=\mathbf{K}_{\mathrm{sh}}+\sum_{i=1}^{n_{\mathrm{st}}}\left(\mathbf{T}_{i}^{T} \mathbf{K}_{\mathrm{st} \cdot i} \mathbf{T}_{i}\right)
$$

where $n_{\mathrm{st}}^{e}$ is the number of elements of the stiffener and $\mathbf{K}_{\mathrm{st} \cdot i}$ and $\mathbf{T}_{i}$ are the global stiffness and transformation matrices of the $i^{\text {th }}$ stiffener as presented in [14]. In equation (8), the stiffness matrix of the shell structure $\mathbf{K}_{\mathrm{sh}}$ is computed based on the following membrane, bending, and shearing components [21,23]:

$$
\mathbf{K}_{\mathrm{sh}}=\mathbf{K}_{m}+\mathbf{K}_{b}+\mathbf{K}_{s}=\iint_{\Omega} \mathbf{B}_{m}^{T} \mathbf{D}_{m} \mathbf{B}_{m} \mathrm{~d} \Omega+\iint_{\Omega} \mathbf{B}_{b}^{T} \mathbf{D}_{b} \mathbf{B}_{b} \mathrm{~d} \Omega+\iint_{\Omega} \mathbf{B}_{s}^{T} \mathbf{D}_{s} \mathbf{B}_{s} \mathrm{~d} \Omega,
$$



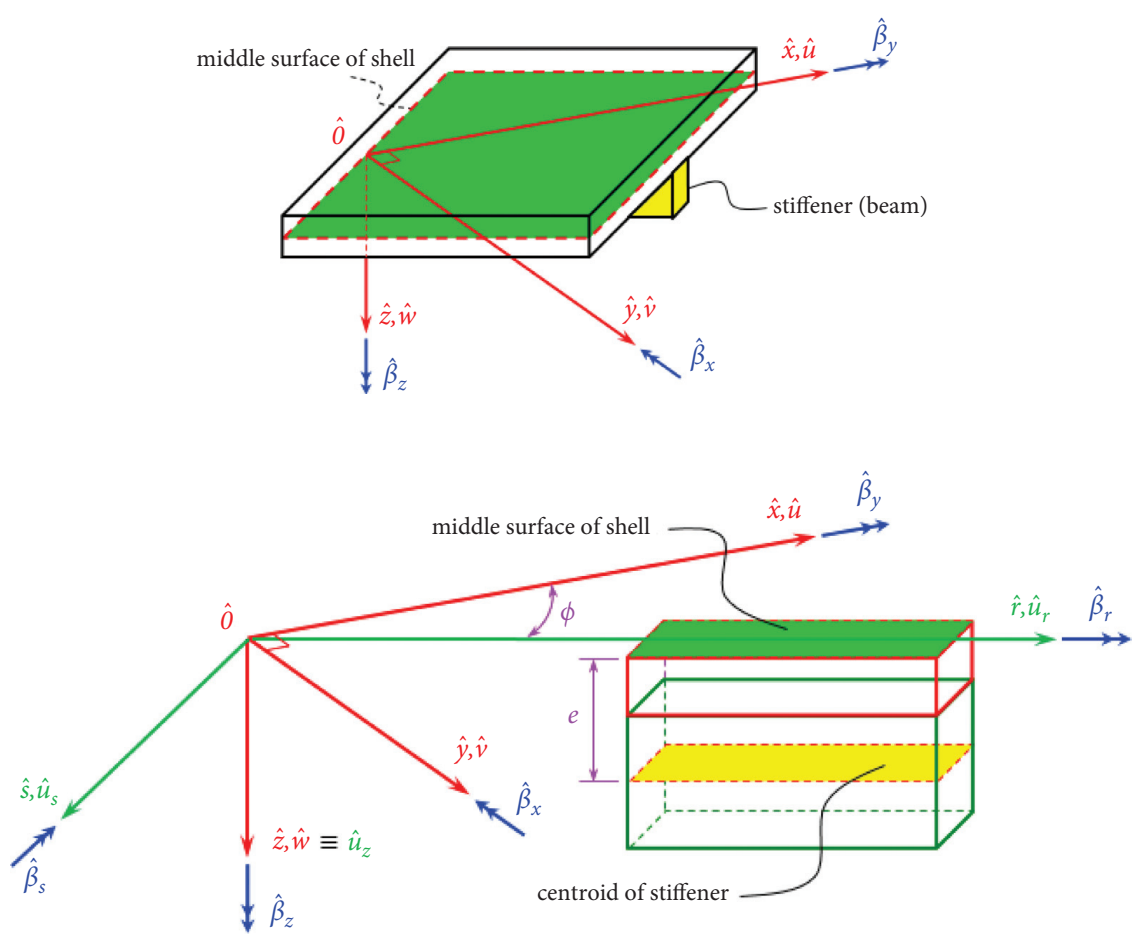

Figure 2: Model of an eccentric stiffener element.

where $\mathbf{B}_{m}, \mathbf{B}_{b}$, and $\mathbf{B}_{s}$ are the FE membrane, bending, and shearing strain gradient matrices [21,23].

In order to avoid the shear-locking phenomenon in the Reissner-Mindlin shell theory, the MITC3 triangular element is applied for the shear strain field in this study. As a result, the shear strain gradient matrix $\mathbf{B}_{s}$ in equation (9) is replaced by the MITC3 shear strain gradient matrix $\breve{\mathbf{B}}_{s}$ as presented in $[21,23]$. The detailed expressions of the MITC3 triangular element can be found in [21,23,24,27].

Recently, an efficient finite element method for shell structure analysis based on a combination of the MITC3 triangular shell element and the edge-based smoothed method (ES-FEM), named as ES-MITC3, is proposed by Pham et al. [21]. Then, the ES-MITC3 is successfully applied to solve the geometrically nonlinear analysis of functionally graded shell structures and transient response of composite shells $[22,23,30]$. In the present paper, the ES-MITC3 method is further extended for the static analysis of stiffened shell structures to enhance the accuracy of the original MITC3 shell elements. By applying the ES-MITC3 method, the stiffness matrix of the shell structure $\mathbf{K}_{\text {sh }}$ in equations (8) and (9) is replaced by a smoothed stiffness matrix $\widetilde{K}_{\mathrm{ES}-\mathrm{MITC} 3}$ [21]. For more detailed information, readers can find in [21].

Finally, to deal with the drill rotation locking issue as discussed in [31], the null values of the stiffness corresponding to the drilling degree of freedom are then replaced by approximate values. This approximate value is taken to be equal to $10^{-3}$ times the maximum diagonal value in the element stiffness matrix [32].

\section{Numerical Results}

In this section, four numerical examples of the stiffened shell are considered to show the high accuracy of the proposed element. For comparison, free shear-locking shell elements such as DSG3 [26], MITC3 [27], and MITC4 [28] are used.

3.1. A Square Plate Stiffened by a Single Beam. We consider a simply supported square plate stiffened by one stiffener as in Figure 3. The plate is subjected to a uniformly distributed load of $q=6.89476 \times 10^{-3} \mathrm{~N} / \mathrm{mm}^{2}$. The material parameters of the plate and stiffened beam are given by Young's modulus $E=1.1721 \times 10^{5} \mathrm{~N} / \mathrm{mm}^{2}$ and Poisson's ratio $v=0.3$. Four uniform meshes of the plate corresponding to $2 \times 2,4 \times 4,6 \times 6$, and $8 \times 8$ elements are used.

The convergence of central displacement for both eccentric and concentric plates using the present ES-MITC3 element is shown in Figures 4 and 5. In these figures, the results based on commercial software NASTRAN [33] and SAP2000 [13] are used as the reference solutions. It can be seen that the results of the ES-MITC3 element present better convergence than those of the MITC3 and DSG3 elements and are virtually equal with those of the superconvergence quadrilateral MITC4 element.

\subsection{A Cantilever Cylinder Shell Stiffened by Concentric Beams.} In this example, the static behavior of a cantilever cylindrical shell stiffened by three concentric stiffeners is investigated. The dimensions, material parameters, and loaded point are shown in Figure 6. Four uniform meshes of the plate 

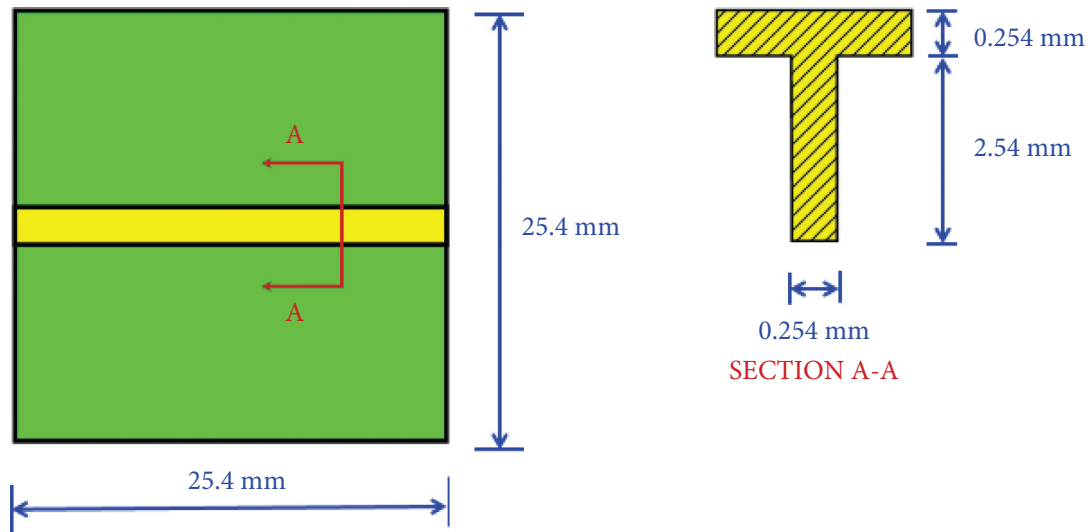

SECTION A-A

Figure 3: A simply supported square plate stiffened by a single stiffener.

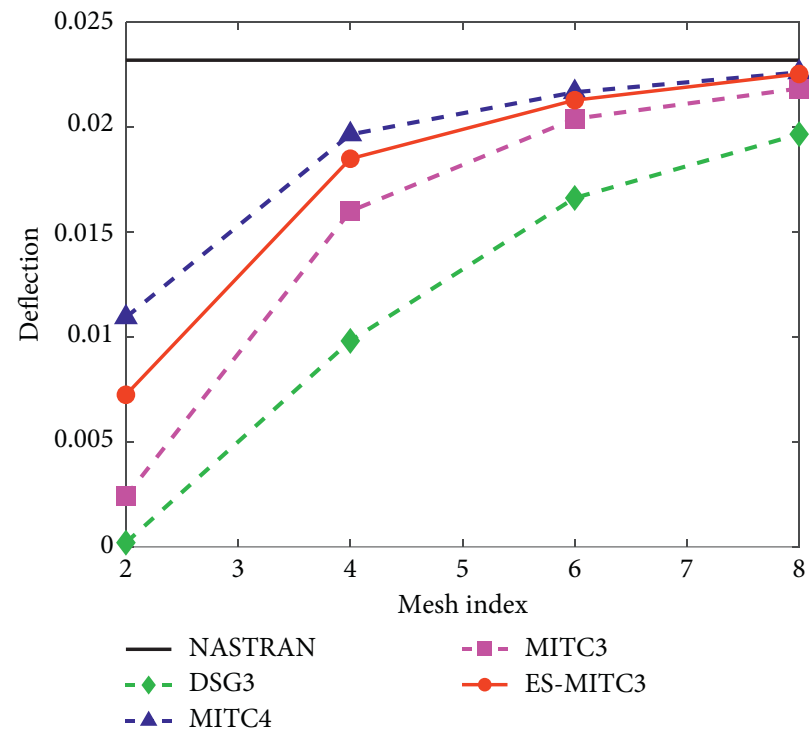

FIGURE 4: The convergence of central displacements of the eccentric stiffened square plate by different methods.

corresponding to $2 \times 2,4 \times 4,6 \times 6$, and $8 \times 8$ elements are also examined. The numerical results of the ES-MITC3 are compared with those of the DSG3, MITC3, and MITC4 elements. In this problem, the results performing by the four-node shell element given by SAP2000 are also used as references, wherein the mesh $12 \times 12$ is used. Figures 7 and 8 show the convergence of the radial and tangential deflections at the loaded point. These figures indicate that the results of the ES-MITC3 are close to the reference solution and converge much better than those of the DSG3, MITC3, and MITC4 elements.

3.3. A Cantilever Cylinder Shell Stiffened by Eccentric and Concentric Beams. We continue to study the stiffened cantilever cylindrical shell subjected to concentrated load. Unlike the previous example when the stiffened cantilever cylindrical used all three concentric stiffeners, in this example, there are two eccentric stiffeners at two the curved edges and only a concentric stiffener at the straight edge of the cylindrical shell, as shown in Figure 9. Figures 10 and 11

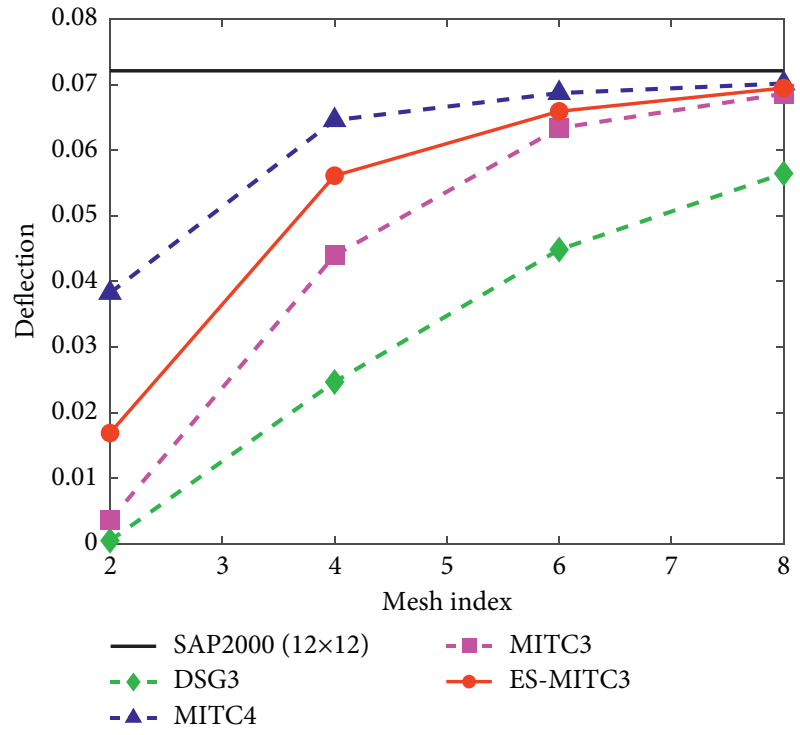

FIGURE 5: The convergence of central displacements of the concentric stiffened square plate by different methods. 

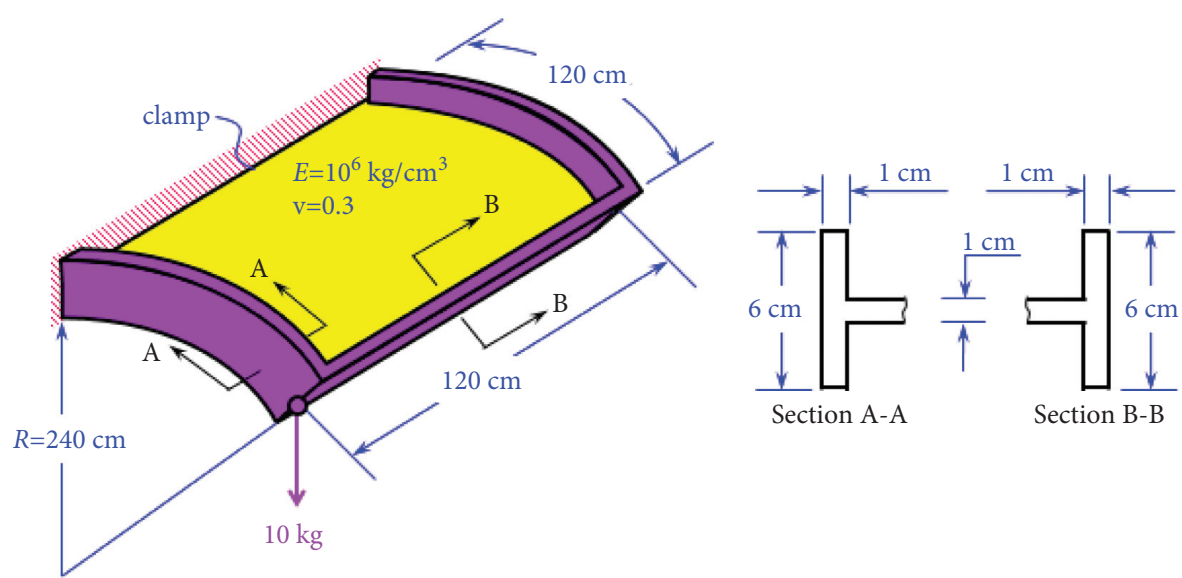

FIGURE 6: Geometry, material parameters, and loaded point of the cantilever cylinder shell stiffened by concentric stiffeners.

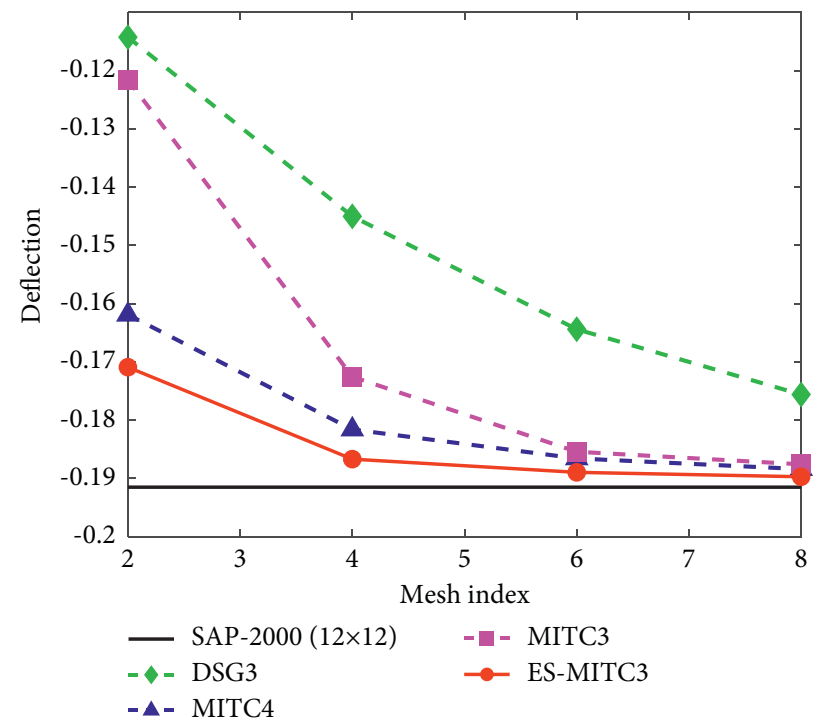

FIGURE 7: The convergence of radial displacements at the loaded point of the concentric stiffened cantilever shell by different methods.

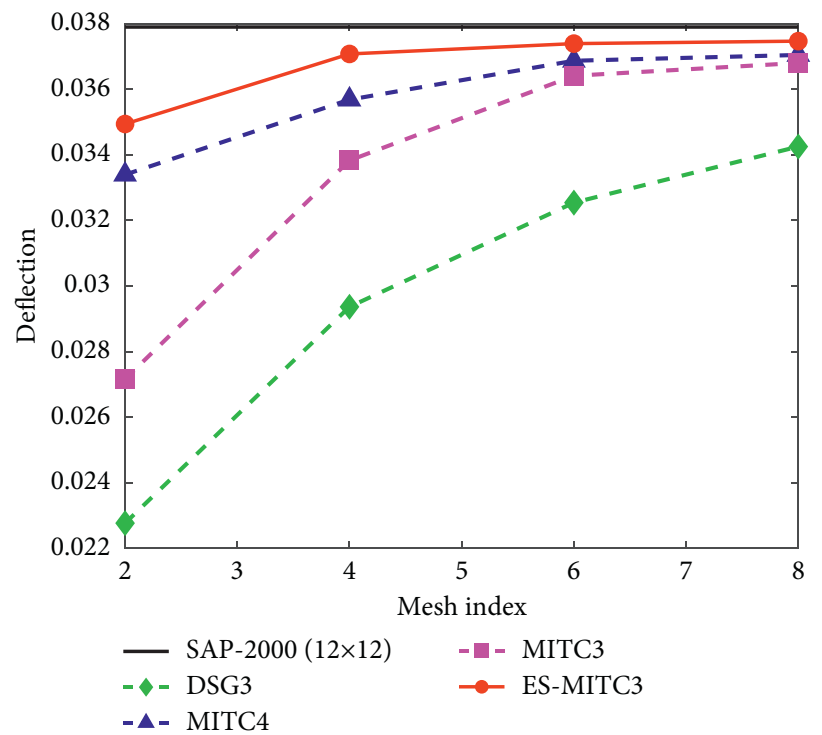

Figure 8: The convergence of tangential displacements at the loaded point of the concentric stiffened cantilever shell by different methods. 


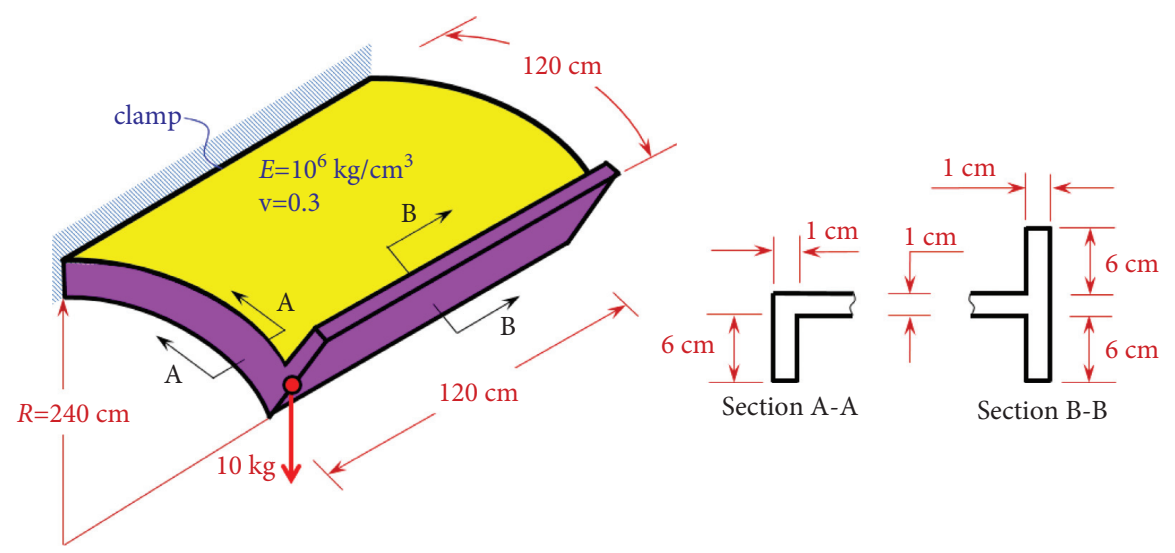

FIGURE 9: Geometry, material parameters, and loaded point of the cantilever cylinder shell stiffened by eccentric and concentric stiffeners.

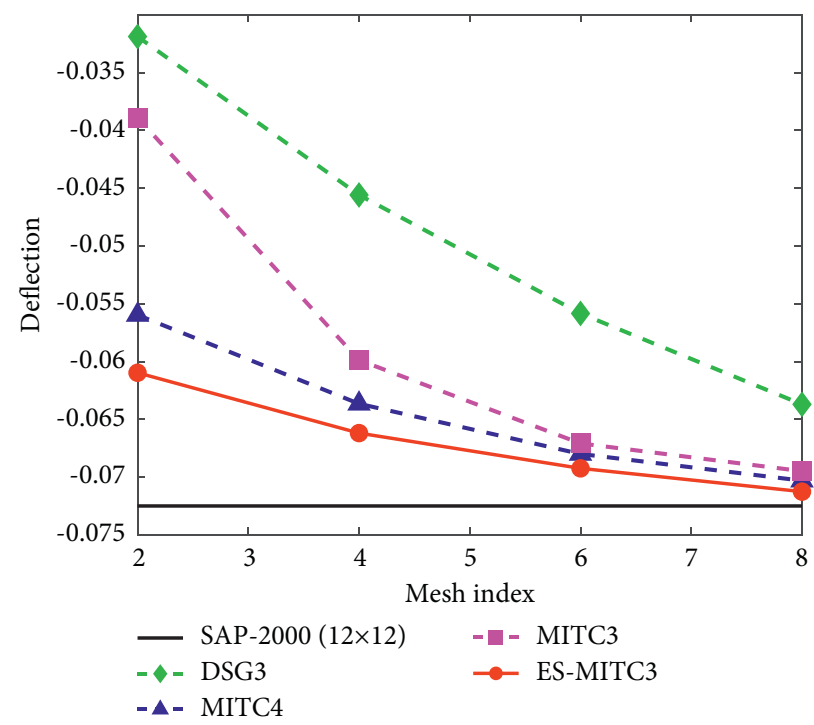

Figure 10: The convergence of radial displacements at the loaded point of the eccentrically stiffened cantilever shell using structured meshes by different methods.

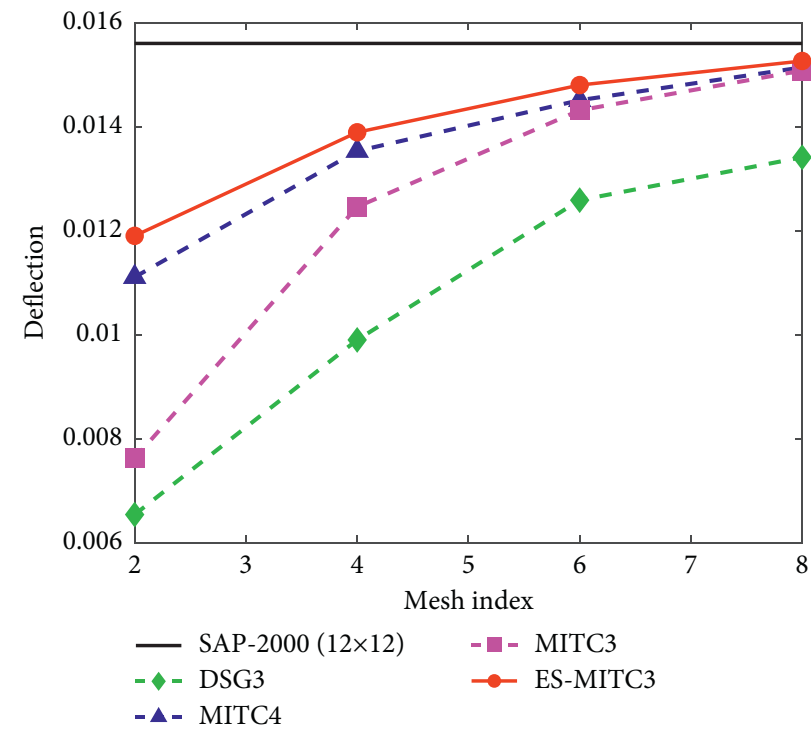

FIGURE 11: The convergence of tangential displacements at the loaded point of the eccentrically stiffened cantilever shell using structured meshes by different methods. 

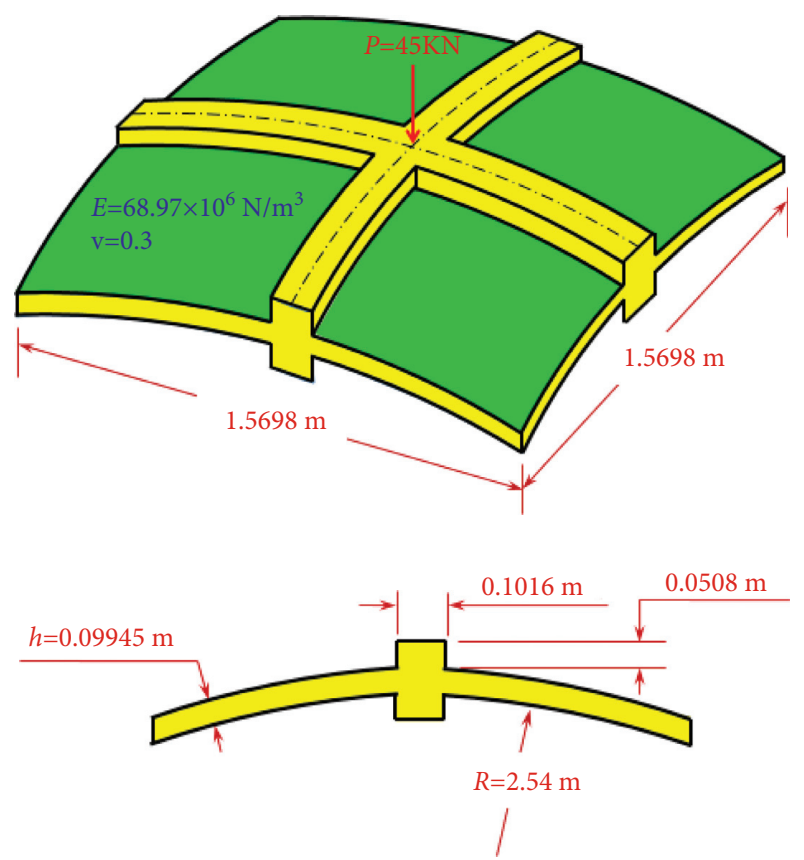

FIGURE 12: The geometry of the stiffened spherical shell panel with simply supported on the boundaries.

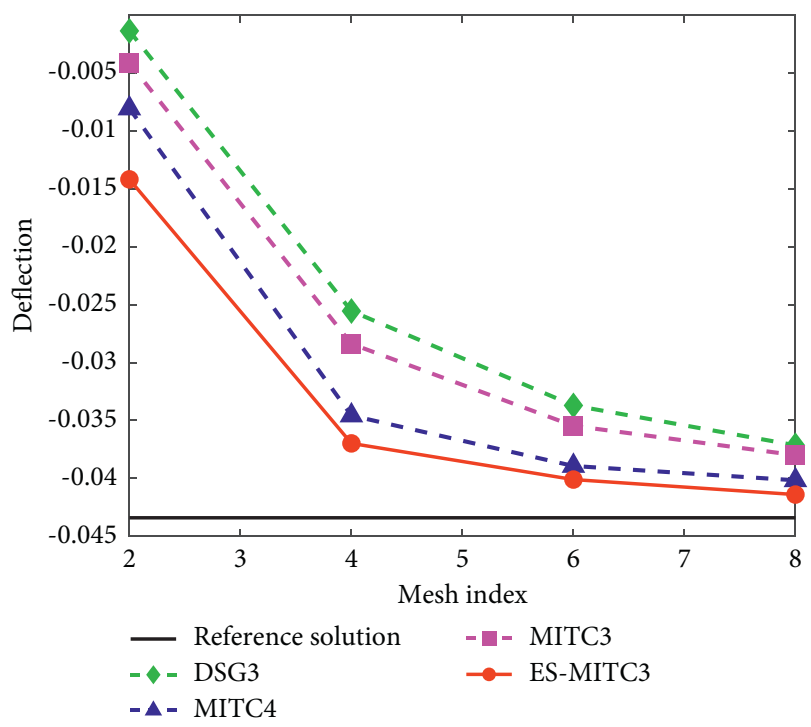

FIGURE 13: The convergence of vertical displacement at the center of stiffened spherical shell panel.

show the convergence of the radial and tangential displacements at the loaded point given by the proposed ESMITC3 element and other elements to the reference solution also given by SAP2000. It can be seen that the results of the proposed method present an excellent performance compared to the MITC3, DSG3, and MITC4 elements.

3.4. Spherical Shell with Cross Stiffeners. Finally, a simply supported spherical shell stiffened by two cross concentric stiffeners is investigated. The dimensions and the material properties of the spherical stiffened shell are shown in
Figure 12. Four uniform meshes of shell corresponding to $2 \times 2,4 \times 4,6 \times 6$, and $8 \times 8$ triangular and quadrilateral elements are used. In this problem, the vertical displacement at the center load is compared, and a reference solution of $w=$ $-0.0434 m$ presented by Prusty and Satsangi [10] with $12 \times 12$ eight-noded isoparametric quadrilateral elements is used. Figure 13 compares the convergence of the vertical deflections at the central load by different methods. It can be again seen that, with the same d.o.f, the triangular shell element ESMITC3 outperforms all other elements, even the MITC4 element, in terms of accuracy and convergence speed.

\section{Conclusion}

This paper presents an extension of the ES-MITC3 for the static analysis of the stiffened shells by combining the original element MITC3 [27] with a linearly isotropic two-node Timoshenko beam element. The compatibility of displacement field of stiffeners and shell is applied at the contact positions. Through the theoretical formulation and numerical results, we can withdraw some following interesting points:

(i) The present ES-MITC3 only uses three-node triangular elements that are much easily generated automatically, even for complicated geometries.

(ii) Owing to the edge-based gradient smoothing operation, the overstiff behavior in the MITC3 [24] has been resolved; hence, the proposed ES-MITC3 significantly improves the accuracy and convergence of the numerical results.

(iii) The ES-MITC3, which only uses the triangular elements, has a clear advantage compared to the fournode quadrilateral elements of MITC4 using the same set of nodes [28].

(iv) For static analysis, the results of the ES-MITC3 agree well with reference results and those by SAP2000. The ES-MITC3 is much more accurate than the DSG3 [26], MITC3 [27], and a good competitor to the MITC4 [28].

\section{Data Availability}

The data underlying the article can be made available or reused.

\section{Conflicts of Interest}

The authors declare that they have no conflicts of interest.

\section{References}

[1] T. P. Holopainen, "Finite element free vibration analysis of eccentrically stiffened plates," Computers \& Structures, vol. 56, no. 6, pp. 993-1007, 1995.

[2] A. Samanta and M. Mukhopadhyay, "Free vibration analysis of stiffened shells by the finite element technique," European Journal of Mechanics - A/Solids, vol. 23, no. 1, pp. 159-179, 2004.

[3] Z. Mecitoğlu, "Vibration characteristics of a stiffened conical shell," Journal of Sound and Vibration, vol. 197, no. 2, pp. 191-206, 1996. 
[4] R. Ruotolo, "A comparison of some thin shell theories used for the dynamic analysis of stiffened cylinders," Journal of Sound and Vibration, vol. 243, no. 5, pp. 847-860, 2001.

[5] Z. Pan, X. Li, and J. Ma, "A study on free vibration of a ringstiffened thin circular cylindrical shell with arbitrary boundary conditions," Journal of Sound and Vibration, vol. 314 , no. 1-2, pp. 330-342, 2008.

[6] W. Flugge, Stress in Shell, Springer, Berlin, Germany, 1st edition, 1934.

[7] G. S. Palani, N. R. Iyer, and T. V. S. R. A. Rao, “An efficient finite element model for static and vibration analysis of eccentrically stiffened plates/shells," Computers \& Structures, vol. 43, no. 4, pp. 651-661, 1992.

[8] A. Venkatesh and K. P. Rao, "Analysis of laminated shells with laminated stiffeners using rectangular shell finite elements," Computer Methods in Applied Mechanics and Engineering, vol. 38, no. 3, pp. 255-272, 1983.

[9] C.-L. Liao and J. N. Reddy, "Analysis of anisotropic, stiffened composite laminates using a continuum-based shell element," Computers \& Structures, vol. 34, no. 6, pp. 805-815, 1990.

[10] B. G. Prusty and S. K. Satsangi, "Analysis of stiffened shell for ships and ocean structures by finite element method," Ocean Engineering, vol. 28, no. 6, pp. 621-638, 2001.

[11] G. Sinha, A. H. Sheikh, and M. Mukhopadhyay, "A new finite element model for the analysis of arbitrary stiffened shells," Finite Elements in Analysis and Design, vol. 12, no. 3-4, pp. 241-271, 1992.

[12] A. Samanta and M. Mukhopadhyay, "Finite element large deflection static analysis of shallow and deep stiffened shells," Finite Elements in Analysis and Design, vol. 33, no. 3, pp. 187-208, 1999.

[13] T. Nguyen-Thoi, T. Bui-Xuan, P. Phung-Van, H. NguyenXuan, and P. Ngo-Thanh, "Static, free vibration and buckling analyses of stiffened plates by CS-FEM-DSG3 using triangular elements," Computers \& Structures, vol. 125, pp. 100-113, 2013.

[14] T. Nguyen-Thoi, T. Bui-Xuan, G. R. Liu, and T. Vo-Duy, "Static and free vibration analysis of stiffened flat shells by a cell-based smoothed discrete shear gap method (CS-FEMDSG3) using three-node triangular elements," International Journal of Computational Methods, vol. 15, no. 06, Article ID 1850056, 2018.

[15] H. Nguyen-Xuan, G. R. Liu, C. Thai-Hoang, and T. NguyenThoi, "An edge-based smoothed finite element method (ESFEM) with stabilized discrete shear gap technique for analysis of Reissner-Mindlin plates," Computer Methods in Applied Mechanics and Engineering, vol. 199, no. 9-12, pp. 471-489, 2010.

[16] H. Nguyen-Xuan, L. V. Tran, T. Nguyen-Thoi, and H. C. Vu-Do, "Analysis of functionally graded plates using an edge-based smoothed finite element method," Composite Structures, vol. 93, no. 11, pp. 3019-3039, 2011.

[17] H. H. Phan-Dao, H. Nguyen-Xuan, C. Thai-Hoang, T. Nguyen-Thoi, and T. Rabczuk, "An edge-based smoothed finite element method for analysis of laminated composite plates," International Journal of Computational Methods, vol. 10, no. 01, Article ID 1340005, 2013.

[18] P. Phung-Van, C. H. Thai, T. Nguyen-Thoi, and H. NguyenXuan, "Static and free vibration analyses of composite and sandwich plates by an edge-based smoothed discrete shear gap method (ES-DSG3) using triangular elements based on layerwise theory," Composites Part B: Engineering, vol. 60, pp. 227-238, 2014.

[19] T. Nguyen-Thoi, T. Bui-Xuan, P. Phung-Van, S. NguyenHoang, and H. Nguyen-Xuan, "An edge-based smoothed three-node mindlin plate element (ES-MIN3) for static and free vibration analyses of plates," KSCE Journal of Civil Engineering, vol. 18, no. 4, pp. 1072-1082, 2014.

[20] T. Nguyen-Thoi, T. Bui-Xuan, P. Phung-Van, S. NguyenHoang, and H. Nguyen-Xuan, "An edge-based smoothed three-node Mindlin plate element (ES-MIN3) for static and free vibration analyses of plates," KSCE Journal of Civil Engineering, vol. 18, no. 4, pp. 1072-1082, 2013.

[21] Q.-H. Pham, T.-V. Tran, T.-D. Pham, and D.-H. Phan, "An edge-based smoothed MITC3 (ES-MITC3) shell finite element in laminated composite shell structures analysis," International Journal of Computational Methods, vol. 15, no. 07, Article ID 1850060, 2017.

[22] D. Pham-Tien, H. Pham-Quoc, T. Vu-Khac, and N. NguyenVan, "Transient analysis of laminated composite shells using an edge-based smoothed finite element method," in Proceedings of the International Conference on Advances in Computational Mechanic, pp. 1075-1094, Phu Quoc Island, Vietnam, August 2017.

[23] Q.-H. Pham, T.-D. Pham, Q. V. Trinh, and D.-H. Phan, "Geometrically nonlinear analysis of functionally graded shells using an edge-based smoothed MITC3 (ES-MITC3) finite elements," Engineering with Computers, vol. 36, no. 3, pp. 1069-1082, 2020.

[24] T. Chau-Dinh, Q. Nguyen-Duy, and H. Nguyen-Xuan, "Improvement on MITC3 plate finite element using edgebased strain smoothing enhancement for plate analysis," Acta Mechanica, vol. 228, no. 6, pp. 2141-2163, 2017.

[25] T.-K. Nguyen, V.-H. Nguyen, T. Chau-Dinh, T. P. Vo, and H. Nguyen-Xuan, "Static and vibration analysis of isotropic and functionally graded sandwich plates using an edge-based MITC3 finite elements," Composites Part B: Engineering, vol. 107, pp. 162-173, 2016.

[26] K.-U. Bletzinger, M. Bischoff, and E. Ramm, "A unified approach for shear-locking-free triangular and rectangular shell finite elements," Computers \& Structures, vol. 75, no. 3, pp. 321-334, 2000.

[27] P.-S. Lee and K.-J. Bathe, "Development of MITC isotropic triangular shell finite elements," Computers \& Structures, vol. 82, no. 11-12, pp. 945-962, 2004.

[28] K.-J. Bathe and E. N. Dvorkin, “A four-node plate bending element based on Mindlin/Reissner plate theory and a mixed interpolation," International Journal for Numerical Methods in Engineering, vol. 21, no. 2, pp. 367-383, 1985.

[29] K. M. Liew, Y. Xiang, S. Kitipornchai, and M. K. Lim, "Vibration of rectangular Mindlin plates with intermediate stiffeners," Journal of Vibration and Acoustics, vol. 116, no. 4, pp. 529-535, 1994.

[30] T. D. Pham, Q. H. Pham, V. D. Phan, H. N. Nguyen, and V. T. Do, "Free vibration analysis of functionally graded shells using an edge-based smoothed finite element method," Symmetry, vol. 11, no. 5, p. 684, 2019.

[31] O. C. Zienkiewicz, R. L. Taylor, R. L. Taylor, and R. L. Taylor, The Finite Element Method: Solid Mechanics, Vol. 2, Butterworth-Heinemann, Oxford, UK, 2000.

[32] T. Nguyen-Thoi, P. Phung-Van, C. Thai-Hoang, and H. Nguyen-Xuan, "A cell-based smoothed discrete shear gap method (CS-DSG3) using triangular elements for static and free vibration analyses of shell structures," International Journal of Mechanical Sciences, vol. 74, pp. 32-45, 2013.

[33] M. P. Rossow and A. K. Ibrahimkhail, "Constraint method analysis of stiffened plates," Computers \& Structures, vol. 8, no. 1, pp. 51-60, 1978. 